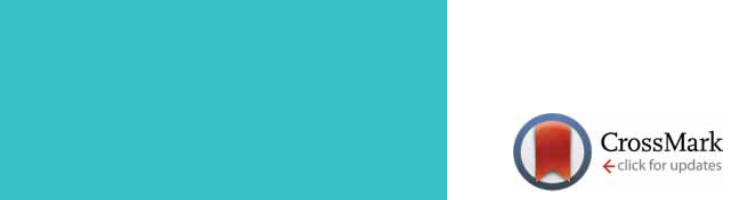

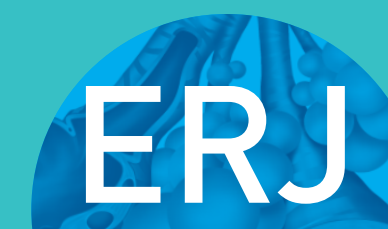

open research
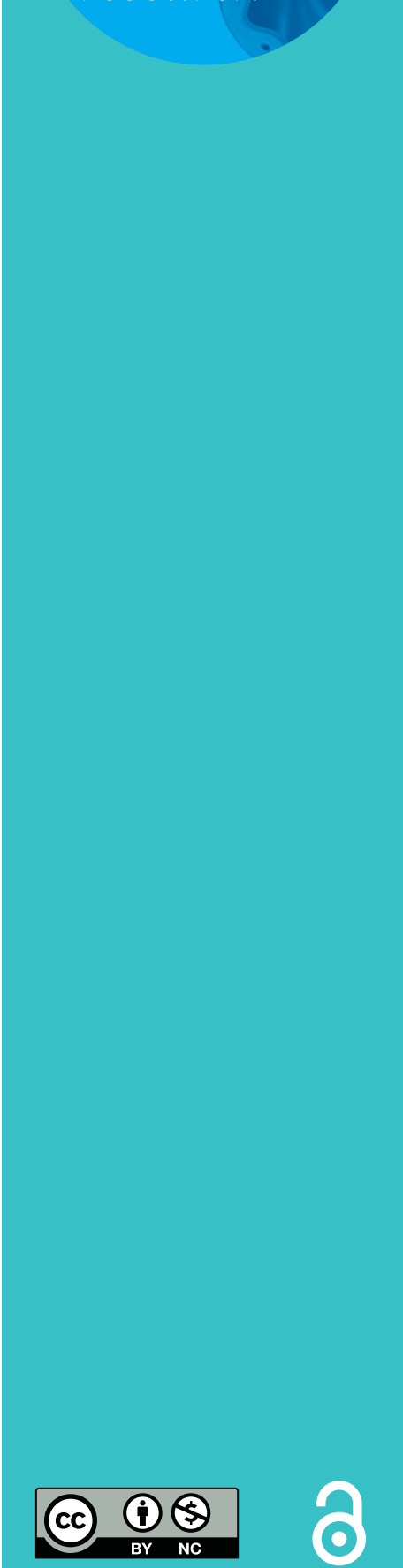

\section{ERS International Congress, Madrid, 2019: highlights from the General Pneumology Assembly}

\author{
Lowie E.G.W. Vanfleteren (1) ${ }^{1}$, Léo Blervaque ${ }^{2}$, Frits M.E. Franssen $\mathbb{1}^{3,4}$, \\ Luke Daines $\mathbb{1}^{5}$, Janwillem W.H. Kocks ${ }^{6,7}$, Persijn J. Honkoop ${ }^{8,9}$ and \\ Vitalii Poberezhets ${ }^{10}$
}

Affiliations: ${ }^{1}$ COPD Center, Sahlgrenska University Hospital and Institute of Medicine, Gothenburg University, Gothenburg, Sweden. ${ }^{2}$ PhyMedExp, INSERM, CNRS, Montpellier University, Montpellier, France. ${ }^{3} \mathrm{CIRO}$, Horn, The Netherlands. ${ }^{4}$ Dept of Respiratory Medicine, Maastricht University Medical Centre, Maastricht, the Netherlands. ${ }^{5}$ Asthma UK Centre for Applied Research, Usher Institute of Population Health Sciences and Informatics, The University of Edinburgh, Edinburgh, UK. ${ }^{6}$ General Practitioners Research Institute, Groningen, The Netherlands. ${ }^{7}$ Observational and Pragmatic Research Institute, Singapore. ${ }^{8}$ Dept of Public Health and Primary Care, Leiden University Medical Center, Leiden, the Netherlands. ${ }^{9}$ Dept of Biomedical Data Sciences, Leiden University Medical Center, Leiden, the Netherlands. ${ }^{10}$ Dept of Propedeutics of Internal Medicine, National Pirogov Memorial Medical University, Vinnytsya, Ukraine.

Correspondence: Lowie E.G.W. Vanfleteren, COPD Center, Sahlgrenska University Hospital and Institute of Medicine, Gothenburg University, Vita Stråket 12, Göteborg 413 45, Sweden. E-mail: lowie.vanfleterendgu.se

ABSTRACT This article contains highlights and a selection of the scientific advances from the European Respiratory Society's General Pneumology Assembly that were presented at the 2019 European Respiratory Society International Congress in Madrid, Spain. The most relevant topics from the different groups will be discussed, covering a wide range of areas including rehabilitation and chronic care, general practice and primary care and M-health and E-health. In this review, the newest research and actual data as well as award-winning abstracts and highlight sessions will be discussed.

@ERSpublications

A highlights review of selected presentations from \#ERSCongress 2019 by the @EuroRespSoc General Pneumology Assembly http://bit.ly/2U2xSjh

Cite this article as: Vanfleteren LEGW, Blervaque L, Franssen FME, et al. ERS International Congress, Madrid, 2019: highlights from the General Pneumology Assembly. ERJ Open Res 2020; 6 : 00323-2019 [https://doi.org/10.1183/23120541.00323-2019].

Received: 20 Nov 2019 | Accepted: 20 Jan 2020

Copyright $\odot$ ERS 2020. This article is open access and distributed under the terms of the Creative Commons Attribution Non-Commercial Licence 4.0. 


\section{Introduction}

The European Respiratory Society International Congress is the world's largest scientific and educational conference in the field of respiratory medicine. For the Madrid 2019 Congress, 4315 abstracts were accepted for presentation. The General Pneumology Assembly of the European Respiratory Society is the largest of the 14 assemblies. In total 317 abstracts were presented in 20 sessions related to this assembly and the four groups of which it consists. It is impossible for any delegate to follow all scientific and clinical advances and breakthroughs presented during this conference. This review aims to provide the reader with an overview of a few of the most interesting presentations of each group, deemed noteworthy by the authors of this manuscript.

\section{Pulmonary rehabilitation and chronic care}

\section{Session: Best abstracts in pulmonary rehabilitation and chronic care}

Work presented in this session covered various important issues in pulmonary rehabilitation (PR), ranging from optimising and maintaining the effects of exercise training to behavioural changes.

Because exercise intolerance often goes hand-in-hand with hypoxaemia in patients with idiopathic pulmonary fibrosis (IPF), devices allowing a higher oxygen flow are of interest. SchNeEberger et al. [1] compared a novel device for supplemental oxygen therapy (SOT), the Oxymizer (Drive DeVilbiss Healthcare, Port Washington, NY, USA), to the conventional nasal cannula (CNC) in 26 IPF patients with SOT indication. 22 patients completed two endurance shuttle walk tests using, in a randomised cross-over design, both Oxymizer or CNC. The use of Oxymizer instead of CNC improved walking capacity and oxygen saturation on pulse oximetry $S_{\mathrm{pO}_{2}}$ and was associated with lower heart and breathing rate. Despite these effects, a majority of patients (64\%) still preferred the CNC for daily use. The authors concluded that offering SOT through Oxymizer could provide clinically relevant benefits for IPF patients.

Additional prognostic information in chronic obstructive pulmonary disease (COPD) patients can be provided with the assessment of functional and exercise capacity, but this assessment is difficult to implement in care context. Thus, WALSH et al. [2] aimed to test the prognosis value of $4 \mathrm{~m}$ gait speed (4MGS) in a cohort of 371 patients with COPD attending hospital outpatient clinics. In a multivariate analysis, they compared the predictive values of $4 \mathrm{MGS}$, Age, forced expiratory volume in $1 \mathrm{~s}\left(\mathrm{FEV}_{1}\right)$ and

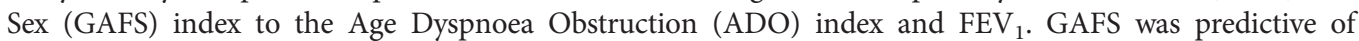
3-year mortality with a better discrimination than the other indexes, with an area under the curve (AUC) of 0.74 . The model was also validated in an independent cohort of 472 patients attending community COPD clinics. The AUC for this cohort was 0.74. The authors concluded that gait speed can provide additional prognostic information than $\mathrm{ADO}$ or $\mathrm{FEV}_{1}$ alone in $\mathrm{COPD}$ patients, in a feasible manner.

DuARTE Freitas et al. [3] conducted a randomised-controlled trial to investigate whether a behavioural change intervention can improve asthma control through an increase of physical activity levels. Control group and intervention group followed the same usual care intervention, with an addition of 8 weeks of behavioural change intervention focused on goal-setting and feedback for the intervention group. The intervention had significant beneficial effects compared to the control group on asthma clinical control, health-related quality of life and physical activity levels. Because improvement in asthma clinical control and physical activity levels were correlated, the authors suggested that beneficial effects of the behavioural change intervention on asthma clinical control were mediated by the increase in physical activity levels.

A randomised double-blind, cross-over study conducted by BONNEviE et al. [4] aimed to investigate whether noninvasive high- and/or low-frequency lumbar transcutaneous electrical nerve stimulation (HF or LF TENS) can improve endurance capacity of COPD patients, as previously shown with intrathecal administration of fentanyl. To meet these objectives, 10 COPD patients performed in a randomised order three constant workload exercises associated with three modalities of stimulation, a sham TENS, an LF TENS at $4 \mathrm{~Hz}$, or a HF TENS at $100 \mathrm{~Hz}$. LF and HF TENS did not induce differential effects compared to the sham condition on dyspnoea, lower limb fatigue and endurance capacity. However, muscle oxygenation tended to be higher in both TENS conditions compare to sham, reflecting, according to the authors, a modulation of the quadriceps muscle activity.

While muscle dysfunction is well described in COPD patients, evidence regarding temporal muscle mRNA responses to both aerobic exercise training (AET) and training cessation (TC) are lacking. LATIMER et al. [5] conducted a trial in 19 COPD patients and 10 healthy controls investigating mRNA response after 1,4 and 8 weeks of AET and 4 weeks after TC. 94 mRNAs involved in the response to AET were quantified with RT-qPCR from muscle biopsies performed at each time. The authors observed a lack of increase in peak oxygen uptake $\left(V^{\prime} \mathrm{O}_{2}\right.$ peak $)$ following exercise training in COPD patients compared to healthy controls. However, muscle mRNA levels were altered in a similar manner in COPD patients and healthy controls in 
response to AET and TC. The authors concluded that skeletal muscle mRNA responsiveness to AET was not blunted and dissociated from the whole-body $V^{\prime} \mathrm{O}_{2}$ peak.

A survival advantage has been described before for COPD patients who completed PR. Extracting data from the Office for National Statistics concerning Wales and England from Jan 2015 to Jan 2017, Evans et al. [6] aimed to identify whether the case-mix severity differences or the effects of PR themselves explained this advantage. 7092 COPD patients were included. 58\% of the patients completed PR and these patients differed from the non-completer group on age, dyspnoea, comorbidity occurrence, walking distance, home oxygen use, smoking status and hospital admission. The mortality rate was lower in the completer group even with adjustment for the parameters described above. This study described a higher cumulative mortality in patients who did not complete PR, (HR 1.42 (95\% CI 1.20-1.67)), after adjustment for confounding factors.

While the beneficial effects of PR in COPD patients are well described, little is known about strategies allowing long-term maintenance of these benefits. BLERVAQue et al. [7] assessed the efficiency of a pragmatic maintenance programme in COPD patients, with a follow-up of 5 years. This programme included weekly supervised exercise training sessions completed with discussion groups and health education. 144 COPD patients were included. The significant benefit provided by the PR on 6-min walk distance (6MWD) remain significant up to 48 months after the PR discharge in the COPD patients involved in the maintenance program. In the same way, the benefits on dyspnoea (MRC scale) and quality of life (VQ11 questionnaire) were maintained up to 5 years and 4 years respectively.

\section{General Practice and Primary care}

Session: Diagnosis and assessment of airway diseases in primary care

There was a wide range of primary care content at the 2019 ERS International Congress. The Primary Care Day focused on infections, vaccinations, and case studies with excellent speakers on day-to-day respiratory cases. Our highlights this year come from the primary care oral presentations on Sunday 29 September, showcasing research into the diagnosis and assessment of airway diseases.

IRINEO GonZÁlEZ et al. [8], explained that spirometry training in Mexico is optional, and rarely performed in primary care. Addressing this, an education programme was delivered to 439 primary care clinicians. Initial testing identified $33 \%$ of participants did not know how to perform spirometry, and $37 \%$ had no equipment. The education programme demonstrated a great improvement in participant knowledge. A second phase of training including certification of spirometry was delivered 10 months later; however, due to high staff turnover, only 70 of the original participants attended. Future research will consider condensing education and certification into one instalment and increasing spirometry availability.

In the Netherlands, spirometry is straightforward to achieve, but the quality compared to American Thoracic Society (ATS)/European Respiratory Society (ERS) standards [9] is unclear. To investigate, vAN DE Hei et al. [10], assessed usefulness and quality of spirometry from 149 primary care patients. 88\% of spirometry readings were considered "clinically useful" by clinicians (two pneumonologists, 15 GPs), meaning they felt able to make a diagnosis from spirometry and available clinical data. However, when compared to the ATS/ERS standards only $13 \%$ of readings met the full criteria. Furthermore, when clinician diagnoses were compared, there was little agreement between the pulmonologists (kappa $=0.38$ (95\% CI 0.27-0.50)), or between general practitioners and pulmonologists (versus pulmonologist 1 kappa $=0.39$ (95\% CI 0.22-0.44); versus pulmonologist 2; kappa $=0.44$ (95\% CI $0.32-0.55)$ ). These data suggest that meeting the ATS/ERS criteria was not necessary for clinicians to feel confident in making a diagnosis, though agreement between pulmonologists and between pulmonologists and GPs was poor.

Even if spirometry quality is sufficient, demonstrating expiratory airflow limitation in asthma can be difficult. Bronchial provocation can be useful in the diagnostic assessment of asthma [11] but is traditionally performed in hospitals [12]. Therefore, Bins et al. [13], investigated the safety and usefulness of community performed bronchial provocation in a Dutch primary care diagnostic centre. 998 patients underwent histamine bronchial provocation with no adverse events. The authors concluded bronchial provocation of adults in the community was safe and feasible, could reduce the number of referrals to secondary care and that under- and over-diagnosis of asthma occurred if bronchial provocation was not used.

Objective tests are also used to monitor asthma control. To monitor children, guidelines recommend measuring lung function routinely and, in some circumstances, Fractional exhaled nitric oxide $\left(F_{\mathrm{ENO}}\right)[14,15]$. Spirometry and $F_{\mathrm{ENO}}$ are used regularly in hospitals but less commonly in primary care. To investigate the value of spirometry and $F_{\mathrm{ENO}}$ for monitoring asthma in primary care children, Lo et al. [16], trained staff in 10 general practices to perform the tests in the UK. Of the 612 children recruited with "GP diagnosed asthma" or "suspected asthma", 575 achieved spirometry and 472 completed $F_{\mathrm{ENO}} .46 \%$ of children with 
available $F_{\mathrm{ENO}}$ and spirometry reported good control (Asthma Control Test (ACT)/Children's Asthma Control Test $(\mathrm{CACT})>19$ ) but had at least one abnormality identified by objective tests. Interestingly, $49 \%$ of the 191 reporting poor control $(\mathrm{ACT} / \mathrm{CACT} \leqslant 19)$, had normal spirometry and $F_{\mathrm{ENO}}<35 \mathrm{ppb}$. These data suggest that assessing asthma in primary care using symptoms, or objective tests in isolation will not provide a full picture of the child.

Two studies considered how to optimise management of adults with COPD. Firstly, BArON et al. [17], investigated asthmatic traits in 3532 individuals with pulmonologist-diagnosed COPD or asthma-COPD overlap (ACO). Over $60 \%$ had at least one "asthma sign". $7 \%$ had a history of asthma, 13\% had reversibility of $\geqslant 12 \%$ and $16 \%$ had atopy. They concluded that asthma traits could help primary care clinicians consider which individuals with COPD are most likely to benefit from inhaled corticosteroids [18]. Secondly, JORDAN et al. [19] presented a prediction model to identify COPD patients at risk of hospitalisation. Age, COPD Assessment Test score, percentage of predicted $\mathrm{FEV}_{1}$, respiratory admissions in the past year, BMI and diabetes were valuable predictors for an individual being admitted within 2 years. The model performed well (c-statistic 0.75 (95\% CI 0.72-0.79) and will be externally validated.

The final abstract investigated the value of the STOP-BANG questionnaire (SBQ) [20] in identifying individuals with obstructive sleep apnoea (OSA). PLANA PEs et al. [21], recruited 565 primary care adults (30-70 years). $38 \%$ scored $\geqslant 3$ on the SBQ and underwent home polysomnography. Of these, $93 \%$ were diagnosed with OSA. The team concluded an SBQ $\geqslant 3$ could be an adequate threshold to screen for OSA in primary care.

Overall, the session highlighted the breadth of research topics and methods used to investigate clinical questions arising from primary care. Learning from researchers across different countries helped address shared goals and challenges arising from efforts to maximise the value of new and existing questionnaires, models and tests to improve the diagnosis and management of respiratory disease.

\section{M-health/E-health}

Session: M-health/e-health poster discussion session

This year the ERS experienced the birth of the special interest Group on m-health/e-health, in the General Pneumology Assembly. This new group actively invites respiratory researchers, scientists, patient organisations, industry representatives, technicians and statisticians with experience in digital technologies to become a part of this movement and to contribute with their specific knowledge. At the m-health/ e-health poster discussion session, we got a marvellous first glimpse of the future of respiratory medicine and were provided with a selected sample of current initiatives.

\section{Telemedicine}

Telemedicine is a very promising field of medicine, especially for hospitals caring for rural areas, because it provides a way to overcome travelling distances. Additionally, it allows for further communication and checks that would not be convenient in a regular setting. During the session, DE LAS HeRAs et al. presented the results of a randomised controlled trial in patients with IPF comparing usual care to tele-rehabilitation with a Virtual Autonomous Physiotherapist Agent (VAPA), on exercise capacity and quality of life [22]. They showed that tele-rehabilitation with VAPA is feasible for IPF and found a significant difference in the 6MWD. VILARINHO et al. presented the results of the introduction of continuous telemonitoring in home mechanical ventilation. In $8 \%$ of patients, they found a clinically relevant situation to act, which could be solved with just a phone call in $87 \%$ of cases [23]. This telemedicine troubleshooting could enhance the compliance of long-term therapy in home respiratory therapies.

Health apps

In recent years, taking into account the growing number of smartphone users, we have seen apps rapidly taking on a central role in people's lives. They can be used to promote self-management, follow up disease outcomes, detect deterioration early and provide information on a variety of issues. At our session, RiJSSENBEEK-Nouwens et al. showed that quality of life and asthma control remained at a higher level in asthma patients after pulmonary rehabilitation if they used an e-health support platform compared to those who received usual care. Interestingly, this was particularly so for high engagers of the e-health system, suggesting that this is an important parameter to measure [24]. PINHeIRo et al. [25] displayed a beautifully created app for children, making use of gamification to increase adherence. Children had to perform a forced expiratory manoeuvre that was registered by their smartphone's microphone. This resulted, if performed properly, in a dragon creating a huge fireball, which blew away a structure and interestingly, if performed sub-optimally, in smaller fireballs. The use of the app meant children performed spirometry unknowingly, and optimal performance was incentivised, in an intuitive and fun way. PUig SANCHEZ et al. [26] presented trial results comparing a newly developed stop smoking app to 
usual care. It included motivational texts upon request and mini-games, which patients could start when they felt the need for a smoke. They showed significantly increased cessation rates, which was all the more impressive considering most patients in the intervention group hardly used the app. A low uptake in app usage was a common feature across different presentations. Future research should focus on user engagement from the start, for example by incorporating end-users in the design phase of the app.

\section{Devices}

The stethoscope is one of the defining features of the doctor, featuring prominently around the neck in movies or television series that include doctor. But what if this stethoscope doesn't actually need the doctor any more? This is the central question that GRZYWALSKI et al. [27] sought to answer by assessing multiple respiratory sounds with plug-in stethoscopes with automatic sound detection and analysis. This automated device was significantly better at detecting different respiratory sounds than doctors. Fortunately, health professionals are still required to interpret the plug-in stethoscope in the context of medical history and other tests. However, it does paint a future whereby the actual presence of the patient inside the hospital might no longer be required for all diagnostics. Another important area of innovation is the emergence of "Quantified Self" and the use of all sorts of wearable devices. MANNÉE et al. [28] presented the first model of an easy to use smart shirt, specifically designed to assess tidal volumes during different types of daily activity. This could be used at home instead of having to come to the clinic for exercise testing.

\section{Artificial intelligence and big data}

Artificial intelligence (AI) and machine learning techniques are of great interest for many involved in $\mathrm{m}$-health. Of course, this presents a very promising new field of medicine, with hopeful reports of earlier detection and more personalised treatment of all sorts of diseases. Demchuk et al. [29] showed an automated decision support system providing personalised treatment advice for pneumonia, taking into account all comorbidities and interactions with other medication. This resulted in significantly fewer medication errors. However, the use of AI in medicine is certainly not a field without controversy. $\mathrm{AI}$ provokes interest as it is potentially superior to existing methods of analysis but also uncertainty as data analysis by neural networking is not well understood. This poses the question: "Should we follow the advice of a system if we do not understand how the system came to that advice?" Interestingly, Das et al. [30] presented a new type of pulmonary function interpretation algorithm that shed some light on which determinants played a role in AI decision making and provided a new score that gives an indication of whether AI is right or not. Hopefully, with further development, we can get more of a grip on how to use $\mathrm{AI}$ in the future and understand it better.

The ERS Congress 2019 was of great interest for those engaged in digital medicine. As well as presentations, the new m-health/e-health Group had its first meeting and shared ideas for next year's conference, to present in the digital health sessions.

\section{Concluding remarks}

The authors of this article hope that this short summary of the impressive amount of lung research and advances in pulmonary care presented through the General Pneumology Assembly of the ERS creates curiosity to follow up on topics of interest to each individual reader.

It was our goal to stimulate discussion and exchange of scientific novelties and clinical developments. We also hope to have encouraged the readership to attend the ERS International Congress 2020 in Vienna.

Conflict of interest: L.E.G.W. Vanfleteren reports grants and personal fees from AstraZeneca, personal fees from Novartis, GSK, Chiesi, Menarini, Pulmonx and Boehringer, and grants from Fisher \&amp; Paykel, outside the submitted work. L. Blervaque reports that as a presenter in the session "Best abstract in pulmonary rehabilitation and chronic care", a part of the submitted work consists of a highlight of his own presentation, but this highlight has been presented neutrally and without any advantage regarding the other presentations. F.M.E. Franssen reports grants and personal fees from AstraZeneca and Novartis, personal fees from Boehringer Ingelheim, Chiesi and GlaxoSmithKline, and personal fees from TEVA, outside the submitted work. L. Daines has nothing to disclose. J.W.H. Kocks reports grants, personal fees and nonfinancial support to his institution from AstraZeneca, Boehringer Ingelheim and GSK, grants and personal fees to his institution from Chiesi Pharmaceuticals and Novartis, and grants from MundiPharma and TEVA, outside the submitted work. P.J. Honkoop reports personal fees from E-wise for making an online course on difficult to manage asthma, outside the submitted work. V. Poberezhets has nothing to disclose.

\section{References}

1 Schneeberger T, Gloeckl R, Jarosch I, et al. Acute effects of supplemental oxygen therapy (SOT) using different nasal cannulas on walking capacity in patients with idiopathic pulmonary fibrosis (IPF) - a randomized cross-over trial. Eur Respir J 2019; 54: Suppl. 63, OA3573. 
2 Walsh JA, Nolan CM, Kon SSC, et al. Gait speed and mortality in chronic obstructive pulmonary disease (COPD). Eur Respir J 2019; 54: Suppl. 63, PA3414.

3 Duarte Freitas P, Passos NFP, Carvalho Pinto RM, et al. Effects of a behaviour change intervention aimed at increasing physical activity on clinical control of adults with asthma: an RCT. Eur Respir J 2019; 54: Suppl. 63, OA3574.

4 Bonnevie T, Gravier F-E, Prieur G, et al. Effects of lumbar transcutaneous electrical nerve stimulation (TENS) on endurance capacity in patients with COPD: a randomized double-blind cross-over study. Eur Respir J 2019; 54: Suppl. 63, OA3575.

5 Latimer L, Constantin D, Popat B, et al. The responsiveness of skeletal muscle mRNAs to aerobic exercise training is not blunted in COPD. Eur Respir J 2019; 54: Suppl. 63, OA3576.

6 Evans RA, Greening NJ, Bolton CE, et al. What influences the survival advantage following pulmonary rehabilitation in patients with COPD? Eur Respir J 2019; 54: Suppl. 63, OA3577.

7 Blervaque L, Préfaut $\mathrm{C}$, Forthin $\mathrm{H}$, et al. A 7-year pulmonary rehabilitation maintenance program: benefits for exercise tolerance, dyspnea and quality of life in patients with obstructive lung disease. Eur Respir J 2019; 54 Suppl. 63, OA3578.

8 Irineo González L, Pérez REB, Garcia JCV, et al. Impact on spirometry knowledge after short training in mexican primary care. Eur Respir J 2019; 54: Suppl. 63, OA251.

9 Miller MR, Hankinson JATS, Brusasco V, et al. Standardisation of spirometry. Eur Respir J 2005; 26: 319-338.

10 van de Hei SJ, Barretta H-J, Doornewaard- ten Hertog NE, et al. Quality of spirometry in primary care: a focus on clinical use. Eur Respir J 2019; 54: Suppl. 63, OA252.

11 Cockcroft DW, Murdock KY, Berscheid BA, et al. Sensitivity and specificity of histamine PC20 determination in a random selection of young college students. J Allergy Clin Immunol 1992; 89: 23-30.

12 Coates AL, Wanger J, Cockcroft DW, et al. ERS technical standard on bronchial challenge testing: general considerations and performance of methacholine challenge tests. Eur Respir J 2017; 49: 1601526.

13 Bins J, Metting EI, Muilwijk-Kroes J, et al. The use of a direct bronchial challenge test in primary care to diagnose asthma: an observational real-life study. Eur Respir J 2019; 54: Suppl. 63, OA253.

14 NICE Guideline. Asthma: Diagnosis, Monitoring and Chronic Asthma Management, NICE nG80 (2017). www. nice.org.uk/guidance/ng80. Date last accessed: November 2019.

15 Global Initiative for Asthma. Global Strategy for Asthma Management and Prevention. 2019. www.ginasthma.org. Date last accessed: November 2019.

16 Lo DK, Beardsmore CS, Roland D, et al. Lung function and asthma control in school-age children managed in UK primary care: a cohort study. Thorax 2019; 75: 101-107.

17 Baron AJ, Blok BF-d, Van EH, et al. Prevalence of asthma characteristics in COPD patients in a Dutch well-established asthma/COPD service for primary care. Eur Respir J 2019; 54: Suppl. 63, OA256.

18 Singh D, Agusti A, Anzueto A, et al. Global strategy for the diagnosis, management, and prevention of chronic obstructive lung disease: the GOLD science committee report 2019. Eur Respir J 2019; 53: 1900164.

19 Jordan R, Fitzmaurice D, Martin J, et al. Development of the Birmingham Lung Improvement Studies (BLISS) prognostic score for COPD patients inprimary care: data from the Birmingham COPD cohort. Eur Respir J 2019, 54: Suppl. 63, OA257.

20 Chung F, Yegneswaran B, Liao P, et al. Stop questionnaire a tool to screen patients for obstructive sleep apnea. Anesthesiology 2008; 108: 812-821.

21 Plana Pes M, Calvera ER, Nieto AT, et al. STOP-BANG: a method to detect obstructive sleep apnea and discriminate for severity in primary care. Eur Respir J 2019; 54: Suppl. 63, OA255.

22 de las Heras JC, Hilberg O, Løkke A, et al. Tele-rehabilitation program in idiopathic pulmonary fibrosis. Eur Respir J 2019; 54: Suppl. 63, PA2232.

23 Vilarinho R, Magalhães T, Silva M, et al. Promotion of adherence to home-mechanical ventilation by Telemonitoring technology: a pilot study. Eur Respir J 2019; 54: Suppl. 63, PA2234.

24 Rijssenbeek-Nouwens LH, Beerthuizen T, Snoeck-Stroband JB, et al. eHealth self-management support after high-altitude climate treatment (HACT) of severe asthma: a randomised controlled trial. Eur Respir J 2019; 54 Suppl. 63, PA2230.

25 Pinheiro C, Viana P, Amaral R. Measurement of respiratory function with a mobile application: comparison with a conventional spirometer and evaluation of usability. Eur Respir J 2019; 54: Suppl. 63, PA2233.

26 Puig Sanchez FG, Hernández LC, Benjumea FN, et al. Use of a mobile App to give up smoking. Results from a clinical trial. Eur Respir J 2019; 54: Suppl. 63, PA2237.

27 Grzywalski T, Szajek M, Hafke-Dys $\mathrm{H}$, et al. Respiratory system auscultation using machine learning - a big step towards objectivisation? Eur Respir J 2019; 54: Suppl. 63, PA2231.

28 Mannée D, De Jongh F, Van Helvoort H. Tidal volumes during tasks of daily living measured with a smart shirt. Eur Respir J 2019; 54: Suppl. 63, PA2228.

29 Demchuk AV, Mostovoy YM. Efficacy of using special software "Optimization of medication choice for treatment of patient with comorbidity" (SS) for management of patients with community-acquired pneumonia (CAP) and cardiovascular disease. Eur Respir J 2019; 54: Suppl. 63, PA2229.

30 Das N, Topalovic M, Raskin J, et al. Explaining predictions of an automated pulmonary function test interpretation algorithm. Eur Respir J 2019; 54: Suppl. 63, PA2227. 